\title{
A NEW METHOD FOR A SIDE-SCATTERING LIDAR TO RETRIEVE THE AEROSOL BACKSCATTERING COEFFICIENT
}

\author{
Zhenzhu Wang ${ }^{*}, 1$, Zongming Tao ${ }^{1}$, Dong Liu ${ }^{1}$, Chenbo Xie ${ }^{1}$, Yingjian Wang ${ }^{1,2}$ \\ ${ }^{1}$ Key Laboratory of Atmospheric optics, Anhui Institute of Optics and Fine Mechanics, Chinese Academy \\ of Sciences, Hefei, Anhui 230031, China \\ ${ }^{2}$ University of Science and Technology of China, Hefei, Anhui 230031, China \\ *Email: zzwang@aiofm.ac.cn
}

\begin{abstract}
The side-scattering lidar based on the CCD camera is a powerful tool to measure the nearground aerosol, which is most interesting for the environmental and meteorological departments. The inversion method for the side-scattering lidar is different from the conventional Fernald method due to the differences in two lidar equations. A new inversion method for the side-scattering lidar to retrieve the aerosol backscattering coefficient is proposed for the first time, which is based on the aerosol backscattering coefficient at the ground as a restriction condition. Numerical simulation is used to analyze the convergence of this new method. Case studies indicate that this new method is reasonable and feasible.
\end{abstract}

\section{INTRODUCTION}

Aerosols are colloids of fine solid particles or liquid droplets suspended in the atmosphere. Atmospheric aerosols have substantial influences on human health [1-2], air quality [3-4] and climate changes [5-6] and their loading has significantly increased. Thus backscattering lidar takes an important place in the studies of the atmospheric environment, air pollution and diffusion with its capable of providing rangeresolved atmospheric profiles continuously. But the backscattering lidar system has a shortcoming in the lower hundreds of meters because of the overlap factor caused by the configuration of the transmitter divergence and the receiver's field of view(FOV) at ranges close to the instrument [7]. This limits the backscattering lidar to be applied to the near-range measurement, especially for the fixed vertical-pointing lidar [8]. Therefore, to study the aerosol properties in haze day a new technique without the overlap factor near the ground would be quite useful. And a new inversion method for the side-scattering lidar to retrieve the aerosol backscattering coefficient is also proposed for the first time, which is based on a prior reference value measured by itself at the ground as a constrained condition.

\section{METHODOLOGY}

In the side-scattering lidar, we receive sidescattering light instead of backscattering light and use a CCD detector instead of a telescope [9]. The side-scattering lidar equation can be written as

$$
P(z, \theta)=\frac{P_{0} K A}{D} \beta(z, \theta) T_{t} T_{r} \Delta \theta
$$

Where $\mathrm{P}(\mathrm{z}, \theta)$ is the received photo electron number for the altitude $\mathrm{z}$ and the scattering angle $\theta$ in pixels, $P_{0}$ is the laser photo electron number, $\mathrm{K}$ is the calibration constant representing the system optical efficiency and A is the effective collecting area of the optics, D is the distance from the CCD to a laser beam, $T_{t}$ and $T_{r}$ and is the total atmospheric (aerosol and molecular) transmittance from the laser to the altitude $\mathrm{z}$ and from the altitude $\mathrm{z}$ along the slant path to the $\mathrm{CCD}$ receiver, respectively. $\mathrm{B}(\mathrm{z}, \theta)$ is the total atmospheric side-scattering coefficient, $\Delta \theta$ is the FOV of a pixel. For the lidar equation, there are two different aspects compared to the backscattering lidar: (I) received signals without range square dependence, (II) no overlap factor. Without the overlap problem, the side-scattering lidar is suitable for detecting the aerosol vertical profile in the near range, especially $[8,10]$.

As to the backscattering lidar, there are two variables -- the aerosol extinction coefficient and the backscattering coefficient. Fernald [11] proposed an iterative inversion solution for the backscattering lidar, which is not suitable for sidescattering lidar due to six unknown variables in the side-scattering lidar equation, i.e. the relative phase functions, the backscattering and extinction coefficients for aerosol and molecules. 
Using the relative aerosol phase function, our group has developed a numerical inversion method for the side-scattering lidar [12]. In order to use the backward integral for reducing inversion errors, the reference point is selected at about $1 \mathrm{~km}$ altitude in the method. However, there is a shortcoming lying in the fact that the aerosol backscattering coefficient value at about $1 \mathrm{~km}$ altitude is not easy to get, especially in the foggy and hazy weather condition, which is usually obtained simultaneously from the backscattering lidar. Fortunately, the aerosol backscattering coefficient value at the ground is found easily even in foggy and hazy weather. So in this letter, using the aerosol backscattering coefficient value at the ground as a restriction condition, we propose an iterative inversion method for the sidescattering lidar to retrieve the aerosol backscattering coefficient based on the backward integral. Based on the previous method in the literature [12], our new inversion procedure can be obtained to get the solution for the aerosol backscattering coefficient profile.

\section{RESULTS}

In order to check the convergence of this new inversion method, numerical simulation has been investigated from the backward and forward integral directions. Supposing there is a simulation aerosol backscatter coefficient profile shown in Fig. 1, then the simulated side-scatter lidar signal can be derived from the sidebackscattering lidar equation (1).

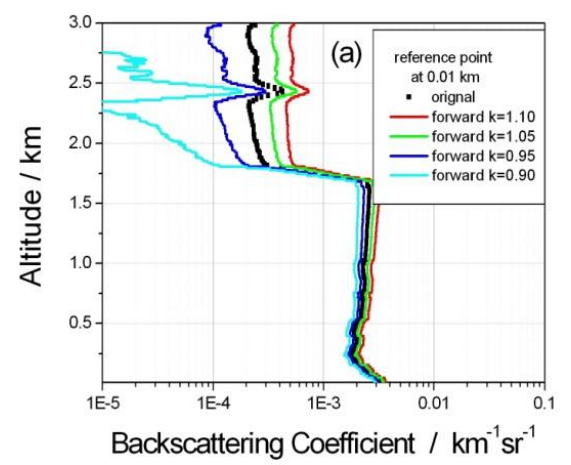

Fig. 1 Retrieval of the aerosol backscattering coefficient profile using the forward integral

As to the forward integral, when we select the reference point at $0.01 \mathrm{~km}$ altitude, the original values of the aerosol backscattering coefficient at the reference point are $0.0033 \mathrm{~km}^{-1} \mathrm{sr}^{-1}$ in Fig.1.
Supposing the aerosol backscattering coefficient is times of the original value at the reference point, and $=1.10,1.05,0.95,0.90$, respectively, the retrieval aerosol backscattering coefficient profiles are calculated in Fig. 1.

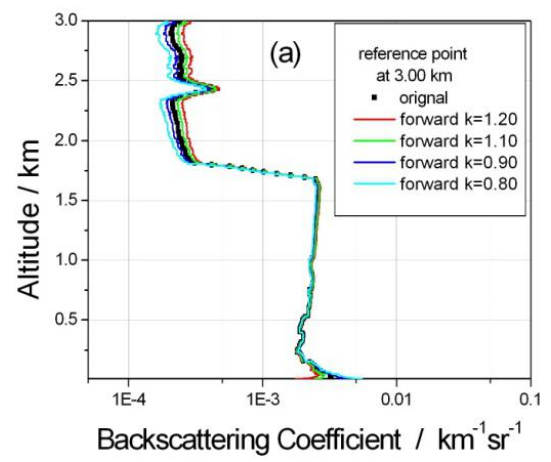

Fig. 2 Retrieval of the aerosol backscattering coefficient profiles using the backward integral

As to the backward integral, the reference point is selected at $3.00 \mathrm{~km}$ altitude, the original values of the aerosol backscattering coefficient at the reference point are $0.00024 \mathrm{~km}^{-1} \mathrm{sr}^{-1}$ in Fig. 2 . Supposing the aerosol backscattering coefficient is times the original values at the reference point, and $=1.20,1.10,0.90,0.80$, respectively, the retrieval aerosol backscattering coefficient profiles are calculated in Fig. 2.

It should be noted that the backward integral will be convergent, and the forward integral cannot be always convergent as shown in Fig. 1 and Fig. 2. As to the forward integral, the bigger the aerosol coefficient and the larger the bias at the reference point, the worse the convergence. The simulation calculations indicate that the backward integral is reasonable direction for the side-scattering lidar inversion.

One measurement by the side-scattering lidar system are presented in the following. In the evening on February 13, 2015, the sky was overcast. The side-scattering lidar and the visibility instrument were operated together at the same place. The visibility instrument was on top of a building at $16 \mathrm{~m}$ altitude. At 19:00, the visibility was $3.87 \mathrm{~km}$, and the corresponding aerosol extinction coefficient was $1.01 \mathrm{~km}-1$ and the corresponding aerosol backscattering coefficient was $0.024 \mathrm{~km}-1 \mathrm{sr}-1$. Taking the aerosol backscattering coefficient $0.024 \mathrm{~km}-1 \mathrm{sr}-1$ at $16 \mathrm{~m}$ altitude as the restriction condition, the 
aerosol backscattering coefficient profile was calculated from the side-scattering lidar equation using the new method, which is shown in Fig. 3 (a)(black line). The red line in Fig. 3(a) was the aerosol backscattering coefficient profile from the previous method [12].

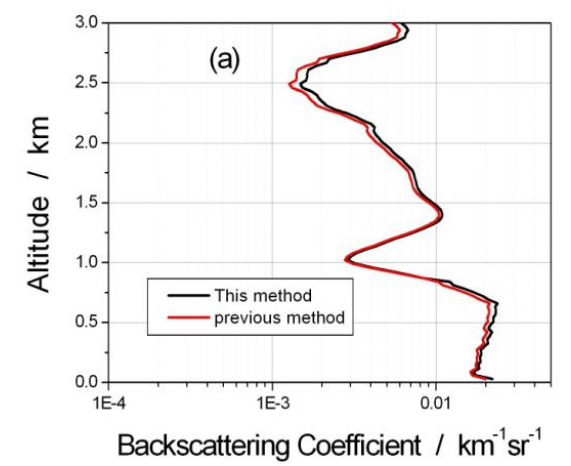

Fig. 3 Retrieved profiles of the aerosol backscattering coefficient from the two methods, at 19:00 LST on February 13, 2015

In summary, a new method for the side-scattering lidar to retrieve the aerosol backscattering coefficient is proposed. This new method solves the important problem: retrieving the aerosol backscattering coefficient without using an expensive backscattering lidar. Numerical simulation and compared experiments indicate that this method is feasible and very suitable to foggy and hazy weather conditions, especially.

\section{ACKNOWLEDGEMENTS}

This work was supported by the National Natural Science Foundation of China under Grant No. 41975038, 41590871, 41811530088 and the Youth Innovation Promotion Association CAS under Grant No. 2017482 and the CAS President's International Fellowship Initiative under Grant No. 2016VEA044.

\section{REFERENCES}

[1] Nel, A. Science, 308(5723), 804-806,(2005).

[2] Zheng, S., et al. Atmos. Chem. Phys., 15(10), 57155725, (2015).

[3] Wang, X., et al. J. Geophys. Res., 115, D00K27, (2010).

[4] Huang, R. J., et al. Nature, 514, 218 - 222,(2014).

[5] Ramanathan, V., et al. J. Geophys. Res., 106(D22), 28371-28398, (2001).

[6] Che, H. Z., et al., Atmos. Chem. Phys., 15, 7619 7652, (2015).

[7] F. Mao, et al. Opt. Laser Technol. 44(4), 907 - 912
(2012).

[8] Z. Wang, et al. Opt. Lett. 40, 1749-1752,(2015).

[9] J. E. Barnes, et al. Appl. Opt. 42(15), 2647-2652, (2003).

[10] Z. Tao, et al. Opt. Express 1127, (2014) .

[11] F. G. Fernald, Appl. Opt. 23(5), 652-653, (1984).

[12] Z. Tao, In Proceeding of International conference on remote senseing, environment and transportation engineering, (Nanjing, China), 250-252,(2013). 